\title{
The Effects of Non-ischemic Fibrosis Texture and Density on Mechanisms of Reentry
}

\author{
Gabriel Balaban ${ }^{1}$, Brian P Halliday ${ }^{2}$, Caroline Mendonca Costa ${ }^{1}$, Bradley Porter ${ }^{1,3}$, Wenjia Bai ${ }^{5}$, \\ Gernot Plank ${ }^{6}$, Christopher A Rinaldi ${ }^{1}$, Daniel Rueckert ${ }^{5}$, Sanjay K Prasad ${ }^{2}$, Martin J Bishop ${ }^{1}$ \\ ${ }^{1}$ School of Biomedical Engineering and Imaging Sciences, King's College London, London, UK \\ ${ }^{2}$ Cardiovascular Research Centre and Cardiovascular Magnetic Resonance Unit, Royal Brompton \\ Hospital, London, UK \\ ${ }^{3}$ Department of Cardiology, Guy's and Saint Thomas Hospital Trust, London, UK \\ ${ }^{4}$ National Heart and Lung Institute, Imperial College, London, UK \\ ${ }^{5}$ Biomedical Image Analysis Group, Deptartment of Computing, Imperial College London, UK \\ ${ }^{6}$ Institute of Biophysics, Medical University of Graz, Austria
}

\begin{abstract}
Patients who present with non-ischemic dilated cardiomyopathy and enhancement on late gadolinium magnetic resonance imaging ( $L G E-C M R$ ), are at high risk of sudden cardiac death. Further risk stratification of these patients based on LGE-CMR may be improved through better understanding of fibrosis micro-structure. Our aim is to examine variations in fibrosis micro-structure based on LGE imaging, and quantify the effect on reentry inducibility.

$2 D$ Computational models were created from a single short axis LGE-CMR image, with variations in fibrosis type (interstitial, replacement) and density. For each fibrosis-type density combination 10 different models were created, each representing a separate random realization. In total 200 models were tested. Reentry inducibility showed a dependence on fibrosis type and density as well as on the specific random realization of the type-density combination.
\end{abstract}

\section{Introduction}

Non-ischemic dilated cardiomyopathy (NIDCM) is characterized by enlarged ventricular cavity size, and impaired ventricular systolic function, which is not as a consequence of myocardial ischaemia. Patients who present with NIDCM are known to be at high risk of sudden cardiac death (SCD), with an estimated $20 \%$ mortality rate over 5 years [1]. Late gadolinium enhanced cardiac magnetic resonance imaging (LGE-CMR) studies of NIDCM have highlighted that approximately one third of NIDCM patients have significant mid-wall fibrosis, corresponding to areas of late gadolinium enhancement (LGE), which are associated with an approximate 4-5-fold increased risk of sudden cardiac death (SCD) [1]. Despite this clear association, the specific physiological processes by which the structural remodelling associated with NIDCM underlies such increased arrhythmic burden remains poorly understood.

Further risk stratification in NIDCM based on LGE-CMR is therefore vital, yet is challenged by the lack of knowledge of both the underlying fibrosis micro-structure, as well as its implications for the generation of reentrant arrhythmia. Based on a single LGE-CMR image, we explored possible variations in fibrosis micro-structure, and determined the consequences for reentry inducibility.

\section{Methods}

\subsection{Image Acquisition and Processing}

An anonymized LGE-CMR image set of a patient presenting with NIDCM and LGE was acquired from the Royal Brompton Hospital, using a previously described protocol [1]. A single short axis (SA) image (Figure 1), with the largest LGE area (semi-automatic segmentation, 3 std $>$ reference mean), was selected. A triangular mesh (max $250 \mu \mathrm{m}$ edge length) of the myocardium was made (CGAL), from smoothed epi, endo and LGE contours, with local myofibre orientations assigned by a LaplaceDirichlet rule based method [2].

\subsection{Fibrosis Microstructures}

Interstitial and replacement fibrosis were modelled by modifying mesh edges and triangles respectively, with a single density parameter $\alpha$ controlling the density of fibrosis in LGE. The probability of fibrosis was related to the 


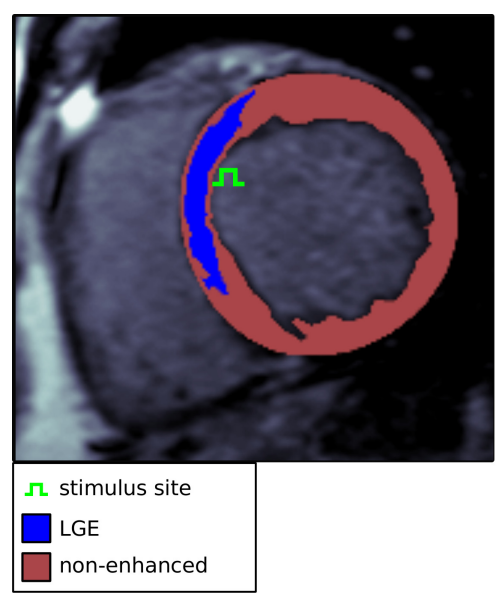

Figure 1. Source late gadolinium enhanced MRI with location of stimulus site and enhanced and non-enhanced areas.

normalized image intensity

$$
I^{*}=\frac{I-I_{\text {ref }}}{I_{\max }-I_{\text {ref }}},
$$

with $I, I_{\max }, I_{r e f}$ denoting the local, maximum, and mean non-LGE reference image intensities respectively, so that higher intensity areas were more likely to contain fibrosis. For replacement fibrosis, the probability of a mesh triangle being fibrotic was

$$
p_{\text {replacement }}=\alpha I^{*},
$$

where $\alpha$ is the global fibrosis density. Fibrotic triangles were removed from the mesh, making them electrically inert and non-conducting.

Interstitial fibrosis was represented by a network of random fibrotic clefts, implemented by doubling mesh verticies across fibrotic edges [3]. Each doubled vertex was assigned to a different neighbouring mesh element along the edge, thereby creating a local no-flux boundary condition aligned with the mesh edge. The probability of an edge being fibrotic was

$$
p_{\text {interstitial }}=\alpha \cos ^{4}(\theta) I^{*},
$$

where $\theta$ is the angle between the triangle edge and the local myocardial fibre direction. Model tissues within circuits of interstitial fibrosis were removed, since they were electrically isolated. Example models with both interstitial and replacement fibrosis at various densities are shown in Figure 2.

\subsection{Conductivities and Conduction Veloci- ties}

Conductivity values in non-enhanced areas were tuned to match conduction velocity (CV) from NIDCM [4], 84

\section{fibrosis texture}

interstitial

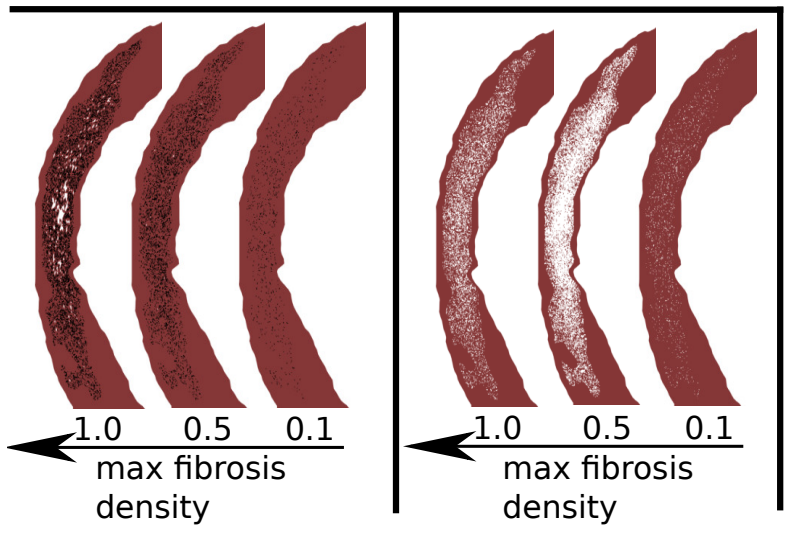

Figure 2. Zoomed-in views of model intersitial and replacement fibrosis patterns with varying maximum fibrosis density.

and $23 \mathrm{~cm} / \mathrm{s}$ in the fibre and transverse directions respectively. Model LGE areas were assigned one of four reduced CVs based on image intensity. Regions in the intensity range $0-25 \%$ and $25-50 \%$ above threshold had transverse conductivity (CVT) reduced by $25 \%$ and $50 \%$ respectively, with normal fibre direction conductivity (CVF). Finally, regions in the intensity ranges $50-75 \%$ and 75 $100 \%$ above threshold had CVF reduced by $25 \%$ and $50 \%$ respectively, and CVT reduced by $50 \%$. These four reduced sets of CV values are consistent with [4], which reported a correlation between fibrosis level and CV slowing, as well as a tendency for preserved longitudinal $\mathrm{CV}$ with milder levels of fibrosis.

\subsection{Electrophysiology Simulation}

Electrical activity was simulated by the standard monodomain formulation, with ionic currents represented by the Ten-Tusscher 2006 model of the human ventricular action potential [5], integrated with step size $20 \mu \mathrm{s}$. Both monodomain solver and cell model are implemented in the Cardiac Arrhythmia Research Package (CARP) [6].

All models were paced from the same site on the LV septal endocardium, approximately halfway down the extent of the LGE (see Figure 1). Stimuli had a square shape with $500 \mu \mathrm{m}$ edge length, strength $500 \mu \mathrm{A} / \mathrm{cm} 2$, and duration $2 \mathrm{~ms}$.

\subsection{Stimulation Protocols}

Reentry inducibility was tested with a dynamic algorithm, with up to six stimuli with variable coupling interval $(\mathrm{CI})$. Each CI was determined by adjusting the timings 
of the next stimulus, and examining whether the stimulus generated a new wave of activation lasting at least $20 \mathrm{~ms}$. The minimum CI which generated a new wave was selected, up to a lower limit of $200 \mathrm{~ms}$.

Reentry was detected if any activations were present after $170 \mathrm{~ms}$ in a circle with radius $3 \mathrm{~mm}$, centered at the stimulus site, and excluding LGE.

\section{Results}

Figure 3 shows the number of reentries per fibrosis typedensity combination. In total 133 reentries were induced, 71 were with interstitial fibrosis, and 62 with replacement fibrosis. For both fibrosis types there was a minimum level of fibrosis density necessary for reentry. These were 0.2 , 0.4 for the interstitial and replacement types respectively.

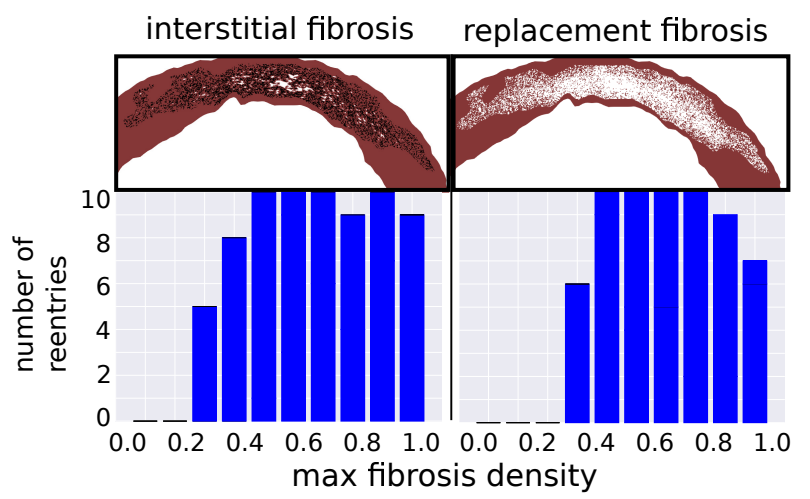

Figure 3. Number of reentries observed out of 10 realizations for each fibrosis density-type combination.

\section{Discussion}

Both fibrosis density and type played a role in the possibility of generating a reentry in our model NIDCM geometry. This is significant because detailed quantification of the absolute type and level of fibrosis in LGE images in NIDCM patients is currently not possible, due to the lack of comparison between core scar and remote tissue, as is commonly used in assessing infarct fibrotic density in patients with ischemic cardiomyopathy [7].

Future developments should address the in-vivo estimation of key fibrosis characteristics, such as density and fibrosis type, as these would greatly improve the predictive capability of computational modelling. This information, when combined with our modelling methodology, has the potential to predict SCD in a manner similar to that which has recently been demonstrated for ischemic disease [8], and thereby pave the way for personalized in-silico prediction of arrhythmic risk in NIDCM.

\subsection{Limitations}

All models were based on a single image and used a single pacing site. Consequentially our results do not account for geometric variability, such as wall thickness, scar transmurality, scar extent as well as pacing location. These factors may affect simulated reentry inducibility.

\section{Acknowledgments}

The authors thank Hermenegild Arevalo for his advice regarding the design of the dynamic stimulation protocol used in our experiments.

\section{Funding}

This work was supported by the National Institute for Health Research Biomedical Research Centre at Guy's and St. Thomas' Trust and King's College, in addition to the Centre of Excellence in Medical Engineering funded by the Welcome Trust and Engineering and Physical Sciences Research Council (EPSRC; WT 088641/Z/09/Z). The views expressed are those of the author(s) and not necessarily those of the National Health Service, and the National Institute for Health Research, or the Department of Health. The authors acknowledge the British Heart Foundation under Project Grant No. PG/14/66/30927. M.J.B. is supported by a Medical Research Council New Investigator Grant (MR/ N011007/1).

\section{References}

[1] Gulati A, Jabbour A, Ismail TF, Guha K, Khwaja J, Raza S, Morarji K, Brown TD, Ismail NA, Dweck MR, et al. Association of fibrosis with mortality and sudden cardiac death in patients with nonischemic dilated cardiomyopathy. Journal of the American Medical Association 2013;309(9):896-908.

[2] Bayer JD, Blake RC, Plank G, Trayanova NA. A novel rulebased algorithm for assigning myocardial fiber orientation to computational heart models. Annals of Biomedical Engineering 2012;40(10):2243-2254.

[3] Costa CM, Campos FO, Prassl AJ, dos Santos RW, SánchezQuintana D, Ahammer H, Hofer E, Plank G. An efficient finite element approach for modeling fibrotic clefts in the heart. IEEE Transactions on Biomedical Engineering 2014; 61(3):900-910.

[4] Anderson KP, Walker R, Urie P, Ershler PR, Lux RL, Karwandee SV. Myocardial electrical propagation in patients with idiopathic dilated cardiomyopathy. The Journal of Clinical Investigation 1993;92(1):122-140.

[5] Ten Tusscher KH, Panfilov AV. Alternans and spiral breakup in a human ventricular tissue model. American Journal of Physiology Heart and Circulatory Physiology 2006; 291(3):H1088-H1100.

[6] Vigmond EJ, Hughes M, Plank G, Leon LJ. Computational 
tools for modeling electrical activity in cardiac tissue. Journal of Electrocardiology 2003;36:69-74.

[7] Glashan CA, Androulakis AF, Tao Q, Glashan RN, Wisse LJ, Ebert M, de Ruiter MC, van Meer BJ, Brouwer C, Dekkers OM, et al. Whole human heart histology to validate electroanatomical voltage mapping in patients with nonischaemic cardiomyopathy and ventricular tachycardia. European Heart Journal 2018;

[8] Arevalo HJ, Vadakkumpadan F, Guallar E, Jebb A, Malamas $\mathrm{P}, \mathrm{Wu} \mathrm{KC}$, Trayanova NA. Arrhythmia risk stratification of patients after myocardial infarction using personalized heart models. Nature Communications 2016;7:11437.

Address for correspondence:

Gabriel Balaban

St. Thomas Hospital, Westminster Bridge Rd, Lambeth, London SE1 7EH, UK

gabriel.balaban@kcl.ac.uk 